Noname manuscript No.

(will be inserted by the editor)

\title{
Advertising a social event in a heterogeneous market
}

Daniela Favaretto - Luca Grosset •

\section{Bruno Viscolani}

\author{
Abstract We study the problem of advertising a social event in a segmented \\ market using different media. The result of such advertising process is the \\ evolution of a goodwill vector. We describe the problem as an optimal control \\ one, assuming that the demand is a concave function of goodwill. We prove \\ the existence of an optimal solution and characterize it using the Pontryagin \\ Maximum Principle. This leads to the analysis of a family of linear program- \\ D. Favaretto \\ Dept. of Management, Ca' Foscari University of Venice, San Giobbe-Cannaregio 837, I-30121 \\ Venezia, Italy \\ E-mail: favaret@unive.it \\ L. Grosset \\ Dept. of Mathematics, University of Padova, Via Trieste 63, I-35121 Padova, Italy \\ E-mail: grosset@math.unipd.it \\ B. Viscolani \\ Dept. of Mathematics, University of Padova, Via Trieste 63, I-35121 Padova, Italy \\ Tel.: +39-049-8271397, Fax: +39-049-8271479 \\ E-mail: viscolani@math.unipd.it
}


ming problems. We provide a more explicit and detailed description of optimal solutions for the case of goodwill linear demand.

Keywords Marketing · Advertising · Optimal Control

AMS Classification (2000): 90B60, 49J15, 90C90

\section{Introduction}

In the paper we study a marketing problem formulated as an optimization model. We focus on a special sort of service, namely a social event, such as a concert, a workshop, a soccer match, ... [1] characterize it as being planned at a fixed time, in a precise place, with a fixed supply capacity, requiring an advertising campaign in order to stimulate as wide a participation as possible, admitting the use of different media (e.g., TV, radio, Internet, newspapers, magazines) for advertising. [8] consider a similar context and stress that the time horizon is short, dissemination of information can be done both through advertising, controlled by the organizers, and through word-of-mouth. The latter authors in a different paper ([9]) consider marketing strategies for a one-time entertainment event, which are a combination of advertising and pricing policies. They distinguish a regular market, with advertising effort and fixed price, from a last-minute market, with no advertising effort and price depending on system conditions.

We build a model for a market of a social event which retains only some of the above mentioned features, disregarding in particular word-of-mouth communication and price dependence; on the other hand we assume that the 
market incorporates a heterogeneous structure, which may be exploited in the advertising process. Hence, we formulate the problem considering a segmented market.

The objective of the event organizers is the profit that has to be maximized. Production, or capacity cost is taken as constant, and may be disregarded. In fact, the organizational costs of a concert, a sport meeting, etc. are totally borne at the event realization date independently of the success of the event (number of tickets sold for it).

We consider the problem of determining advertising efforts for selling tickets for a social event in a heterogeneous market, which has been segmented. The relevance of targeting advertising to segments of consumers within a market has been the object of study by [7], who stress the fact that targeted advertising is now specially prominent because of new features of the marketing environment. The decision on advertising in a segmented market has been also studied by $[15]$ and $[2,3]$.

A social event may be considered as a special kind of seasonal product, and the results presented in this paper complement the analysis done by [5]. In that paper the effect of advertising on each segment goodwill was represented by a strictly concave function of the effective advertising effort, and the demand in each segment was represented by a linear function of segment goodwill. Here, the effect of advertising on each segment goodwill is represented by a linear function of advertising effort, and the demand in each segment is represented by a linear or concave function of segment goodwill. Moreover here we consider 
the possibility of using jointly several advertising media with different segment spectra.

The paper is organized as follows. In Section 2 we present the model of a segmented market, where a product goodwill evolves à la Nerlove-Arrow, driven by suitable advertising actions; these are realized by means of different media. In Section 3 we formulate the optimal control problem of advertising a social event, where demand (for tickets) is a concave function of goodwill. In Section 4 we prove the existence of an optimal solution and characterize it using the Pontryagin Maximum Principle. We obtain a more explicit description of optimal solutions for the case of goodwill linear demand, in Section 5. Finally, in Section 6 we comment on the results obtained and provide some hints for possible future developments.

\section{Goodwill evolution in a segmented market}

We want to consider the optimal control problem of determining advertising efforts for maximizing the profit from a social (entertainment) event in a heterogeneous market.

In order to address such a kind of market it is important to segment it. As Easey says, "Market segmentation is where the larger market is heterogeneous and can be broken down into smaller units that are similar in character." [4, p. 253]. Other authors observe that " $\ldots$ a range of media $\ldots$ are used that are said to be targeted specifically at certain segments of the market" ([16]). 
While discussing the success of segmentation of particular media, the same authors stress that media “... play a vital role in the success of targeting efforts." Typical segmentations are obtained using such attributes as region, age, gender, occupation, generation, lifestyle, occasions, ... [12, p. 288].

Let the consumer (or potential attendees) population be partitioned into $n$ groups (segments), each one specified by the value $i \in\{1,2, \ldots n\}$ of a suitable parameter (segmentation attribute). Let $G_{i}(t)$ represent the stock of goodwill of the service/product at time $t$, for the consumers in the $i$ th segment. We refer to the definition of goodwill given by [13] to describe the variable which summarizes the effects of present and past advertising on the demand. The goodwill needs an advertising effort to increase, while it is subject to a spontaneous decay. Here we assume that the goodwill evolution satisfies the set of independent ordinary differential equations

$$
\dot{G}_{i}(t)=w_{i}(t)-\delta_{i} G_{i}(t), \quad i=1, \ldots n
$$

where $\delta_{i}>0$ represents the goodwill depreciation rate for the members of the consumer group $i$ and $w_{i}(t)$ is the effective advertising intensity at time $t$ directed to that same group. For each fixed value of the parameter $i \in$ $\{1, \ldots n\}$, i.e. for each segment, the dynamics of the goodwill given by (1) is the same as the one proposed in [13]. Here, consistently with the assumption of distinct goodwill variables for different market segments, we further assume that both the advertising intensity and the goodwill decay parameter may depend on the attribute value $i$. 
In the following we will write the motion equations (1) in vector notation:

$$
\dot{\boldsymbol{G}}(t)=-\operatorname{diag}(\boldsymbol{\delta}) \boldsymbol{G}(t)+\boldsymbol{w}(t)
$$

where $\operatorname{diag}(\boldsymbol{\delta})$ is the diagonal matrix with diagonal entries the elements of $\boldsymbol{\delta}=\left(\delta_{1}, \ldots \delta_{n}\right)$

We consider an event organizer who bears the organizational costs of the event (a concert, a sport meeting, etc. ) in the time interval $[0, T], T>0$, and will observe the success of the event (number of tickets sold for it) at the final time $T$.

The value of the goodwill vector at the initial time 0 is a known datum,

$$
G(0)=G^{0} \geq 0
$$

where the possible positivity of some components is explained as residual goodwill from past experience with the same event organizer.

The targeting effort in a segmented market, at a given time $t$, is represented by the direction of the advertising effort vector $\boldsymbol{w}(t)$, i.e. by $\|\boldsymbol{w}(t)\|^{-1} \boldsymbol{w}(t)$. In order to choose a specific target, or advertising effort direction, the event organizer wants to use a range of media with specific segment targets $([16])$.

We assume that the organizer may use $m \geq 2$ advertising media which reach different segments with variable effectiveness. Let $\boldsymbol{u}(t) \in[0,+\infty)^{m}$ be the $m$-dimensional advertising effort: its $j$ th component $u_{j}(t)$ is the medium $j$ advertising effort, and we assume that the consequent effective advertising intensity at time $t \in[0, T]$ directed to segment $i$ is

$$
w_{i}^{j}(t)=\gamma_{i j} u_{j}(t), \quad i=1, \ldots n, \quad j=1, \ldots m
$$


for some medium and segment specific parameters $\gamma_{i j} \geq 0$, such that $\sum_{i=1}^{n} \gamma_{i j}>$

0 . We assume that the total effective advertising intensity at time $t$ directed to segment $i$ is $w_{i}(t)=\sum_{j=1}^{m} \gamma_{i j} u_{j}(t), i=1, \ldots n$, so that, in vector notation, we have

$$
\boldsymbol{w}(t)=\boldsymbol{\Gamma} \boldsymbol{u}(t)
$$

where $\Gamma=\left(\gamma_{i j}\right) \in M_{n \times m}(\mathbb{R})$. We call medium $j$ segment-spectrum the $j$ th column $\gamma_{\cdot j}$ of matrix $\boldsymbol{\Gamma}$. Equation (5) represents the additive advertising effects assumption, which is a standard one (see e.g. [10, p. 108], [6], [11]).

\section{Advertising a social event}

We want to determine the optimal advertising policy in the time interval $[0, T]$, in order to maximize the net profit. As the typical advertising period for such events is short (see [8]), we consider undiscounted costs and revenue.

The demand rate in each segment $i$ and time $t$ depends on the goodwill $G_{i}(t)$, through a demand function $f_{i}(\cdot)$, so that the cumulative demand at time $t, x(t)$, is the solution to the Cauchy problem

$$
\dot{x}(t)=\sum_{i=1}^{n} f_{i}\left(G_{i}(t)\right), \quad x(0)=0 .
$$

where $f_{i}(\cdot)$ is a nonnegative, increasing and concave function, with $f_{i}(0)=0$. Its value depends on the dimension of the segment, i.e. number of potential attendees in it, and on the interest of those potential attendees to the event, as represented by the goodwill $G_{i}$. We further assume that it is twice continuously differentiable, with $f_{i}^{\prime}(\cdot)>0$ and $f_{i}^{\prime \prime}(\cdot) \leq 0$. 
The goodwill evolution is driven by the advertising efforts (the control functions) $u_{i}(t) \geq 0$ with constant marginal costs $k_{i}>0$. We define the row vector $\boldsymbol{k}=\left(k_{1}, k_{2}, \ldots k_{m}\right) \in \mathbb{R}^{m}$, so that the total advertising cost rate at time $t$ is the scalar product $\boldsymbol{k u}(t)$.

Let the profit, gross of advertising costs be represented by $R(x)$, where $x$ is the event participation or number of tickets sold, and the constant production cost is disregadred. We assume that $R(\cdot)$ is a nonnegative, increasing and strictly concave function, with $R(0)=0$; we further assume that it is twice continuously differentiable, with $R^{\prime}(\cdot)>0$ and $R^{\prime \prime}(\cdot)<0$. The advertising problem of maximizing the overall profit

$$
J(\boldsymbol{u})=R(x(T))-\int_{0}^{T} \boldsymbol{k} \mathbf{u}(t) d t
$$

subject to the conditions $(2),(5),(6),(3)$, and the control constraints

$$
\begin{gathered}
\boldsymbol{k u}(t) \leq b, \\
\mathbf{0} \leq \boldsymbol{u}(t) \leq \overline{\boldsymbol{u}},
\end{gathered}
$$

for some $\overline{\boldsymbol{u}}$, with $\bar{u}_{j}>0, j=1, \ldots m$, and $b>0$, is an optimal control problem. The upper bound $b$ on the global advertising cost rate $\boldsymbol{k} \boldsymbol{u}(t)$ represents an $a$ priori decision of the event organizer; the same reason, and also technological or contractual reasons justify the upper bounds $\bar{u}_{j}$ on the medium $j$ activation level, for all $j$. 


\section{Optimal solution}

The Hamiltonian function associated with the advertising problem is

$$
H(\boldsymbol{G}, x, \boldsymbol{u}, \boldsymbol{p}, q)=-\boldsymbol{k u}+\boldsymbol{p}(\boldsymbol{\Gamma} \boldsymbol{u}-\operatorname{diag}(\boldsymbol{\delta}) \boldsymbol{G})+q \sum_{i=1}^{n} f_{i}\left(G_{i}\right)
$$

where the row vector $\boldsymbol{p} \in \mathbb{R}^{n}$ and $q \in \mathbb{R}$ are the adjoint variables associated with $\boldsymbol{G}$ and $x$. We observe that the Hamiltonian is a linear function of $(\boldsymbol{G}, x, \boldsymbol{u})$. Using the Pontryagin Maximum Principle (see e.g. [14, p. 182]) we obtain that, if $\left(\boldsymbol{u}^{*}(t), \boldsymbol{G}^{*}(t), x^{*}(t)\right)$ is an optimal solution to the advertising problem, then, for all $t \in[0, T], \boldsymbol{u}^{*}(t)$ is an optimal solution to the linear programming problem

$$
\begin{aligned}
& \operatorname{maximize}(\boldsymbol{p}(t) \boldsymbol{\Gamma}-\boldsymbol{k}) \boldsymbol{u}, \\
& \text { subject to } \quad \boldsymbol{k u} \leq b, \\
& \boldsymbol{u} \leq \overline{\boldsymbol{u}}, \\
& \boldsymbol{u} \geq \mathbf{0} ;
\end{aligned}
$$

for all $i=1,2, \ldots n$, the $i$ th component of the $n$-dimensional adjoint function $\boldsymbol{p}(t)$ satisfies the linear differential equation

$$
\dot{p}_{i}(t)=\delta_{i} p_{i}(t)-q(t) f_{i}^{\prime}\left(G_{i}(t)\right)
$$

and the transversality condition

$$
p_{i}(T)=0 ;
$$

whereas the adjoint function $q(t)$ is constant and has the value

$$
q(t)=q=R^{\prime}(x(T))>0 .
$$


An implicit solution to equation (12) satisfying (13), i.e. with $p_{i}(1)=0$, is

$$
p_{i}(t)=q \int_{t}^{T} e^{-\delta_{i}(s-t)} f_{i}^{\prime}\left(G_{i}(s)\right) d s
$$

The feasible region of the linear programming problem (11) is invariant with respect to time $t$ and it is non-empty and bounded, hence, for all $t \in[0, T]$, the problem (11) has an optimal solution at some vertex of its feasible region.

If there exists a solution to the necessary PMP conditions of the advertising problem, then we can state that it is optimal, using the Mangasarian sufficiency theorem (see e.g. [14, p. 182]), because the Hamiltonian (10) is concave in $(\boldsymbol{G}, x, \boldsymbol{u})$, and $R(x)$ is concave in $x$.

On the other hand, we can state that the advertising problem has an optimal solution, as the assumptions of the existence theorem of Filippov-Cesari [14, p. 132] are satisfied:

- the control $\boldsymbol{u}(t) \equiv \mathbf{0}$ induces an admissible solution;

- the range of the control function is the convex polyhedron

$$
U=\{\boldsymbol{u} \mid \boldsymbol{k u} \leq b, \boldsymbol{u} \leq \overline{\boldsymbol{u}}, \boldsymbol{u} \geq \mathbf{0}\}
$$

a closed and bounded set;

- the objective functional can be written, in Lagrange form, as

$$
J=\int_{0}^{T}\left\{R^{\prime}(x(t)) \sum_{i=1}^{n} f_{i}\left(G_{i}(t)\right)-\boldsymbol{k u}(t)\right\} d t
$$

and therefore the set

$$
\left\{\left(R^{\prime}(x) \sum_{i=1}^{n} f_{i}\left(G_{i}\right)-\boldsymbol{k} \boldsymbol{u}+\xi, \boldsymbol{\Gamma} \boldsymbol{u}-\operatorname{diag}(\boldsymbol{\delta}) \boldsymbol{G}, \sum_{i=1}^{n} f_{i}\left(G_{i}\right)\right), \xi \leq 0, \boldsymbol{u} \in U\right\}
$$


is convex for each $(x, \boldsymbol{G}, t)$, as it is an affine transformation of the $(m+1)$ -

dimensional convex set $(-\infty, 0] \times U$;

- for all admissible control-state functions $(\boldsymbol{u}(t), \boldsymbol{G}(t), x(t))$,

$$
\|(\boldsymbol{G}(t), x(t))\| \leq\left\|\left(\boldsymbol{G}^{0}+T \boldsymbol{\Gamma} \overline{\boldsymbol{u}}, \sum_{i=1}^{n} f_{i}\left(G_{i}^{0}+T \boldsymbol{\gamma}_{i} \cdot \overline{\boldsymbol{u}}\right)\right)\right\|,
$$

where $\gamma_{i}$. is the $i$ th row of the matrix $\boldsymbol{\Gamma}$.

An elementary observation on the problem (11) reveals for an optimal solution $\boldsymbol{u}^{*}$ that

$$
(\boldsymbol{p}(t) \boldsymbol{\Gamma}-\boldsymbol{k})_{j}=\boldsymbol{p}(t) \boldsymbol{\gamma}_{\cdot j}-k_{j}<0 \Rightarrow u_{j}^{*}=0
$$

or that

$$
u_{j}^{*}>0 \Rightarrow \boldsymbol{p}(t) \boldsymbol{\gamma}_{\cdot j}-k_{j} \geq 0
$$

The adjoint functions $p_{j}(t)$ are continuous, with $p_{j}(T)=0$, hence the coefficients of the objective function of the problem (11), i.e. $\boldsymbol{p}(t) \boldsymbol{\gamma}_{\cdot j}-k_{j}$, $j=1,2, \ldots m$, are continuous functions of $t$, and they are negative in a neighborhood of the final time $T$, because

$$
\boldsymbol{p}(T) \boldsymbol{\gamma}_{\cdot j}-k_{j}=-k_{j}<0 \text {. }
$$

As a consequence, any optimal advertising policy $\boldsymbol{u}^{*}(t)$ must be $\mathbf{0}$ in a neighborhood of the final time.

At any given time $t<T$, the optimal solutions to problem (11) are all the points in the convex hull of the optimal vertices of the feasible region.

As a general comment on the linear programming problem constraints, we single out four distinct situations that may occur: 
i) if $b<\min _{j} k_{j} \bar{u}_{j}$, then all the medium activation level constraints are redundant, as they result inactive at all feasible points, and the only relevant constraint is that concerning the global advertising cost rate;

ii) if $\min _{j} k_{j} \bar{u}_{j} \leq b<\max _{j} k_{j} \bar{u}_{j}$, then several medium activation level constraints are relevant, together with the global advertising cost rate constraint;

iii) if $\max _{j} k_{j} \bar{u}_{j} \leq b \leq \boldsymbol{k} \overline{\boldsymbol{u}}$, then all the constraints stated in (11) are relevant;

iv) if $b>\boldsymbol{k} \overline{\boldsymbol{u}}$, then the global advertising cost rate constraint is redundant, and all the constraints concerning the medium activation levels are relevant.

In the extreme situations i) and iv) the feasible region of the problem (11) has particular simple structures and some features of optimal solutions emerge easily.

Although the present analysis has been developed assuming $m \geq 2$, when "several media" makes sense, everything holds also in the degenerate case that $m=1$ and one wants to determine the optinal advertising effort using a single medium. In such a case an optimal control is of the bang-bang type, with values in $\{0, \min \{\bar{u}, b / k\}\}$, where $k$ and $\bar{u}$ are the marginal cost and the maximum admissible activation level of the unique advertising medium.

4.1 Constraint on global advertising cost rate only

Let $b<\min _{j} k_{j} \bar{u}_{j}$, so that we can ignore all the medium activation level constraints, which are redundant, and the only relevant constraint is that concerning the global advertising cost rate. 
The feasible region of the linear programming problem (11), under the present assumption, is characterized by the inequalities

$$
\boldsymbol{k} \boldsymbol{u} \leq b, \quad \boldsymbol{u} \geq \mathbf{0}
$$

it is the $m$-dimensional convex polyhedron with the $m+1$ vertices

$$
\mathbf{0}, \quad \text { and } \boldsymbol{u}^{(j)}=\frac{b}{k_{j}} \boldsymbol{e}_{j}, j=1,2, \ldots m
$$

where $\boldsymbol{e}_{j}$ is the $j$ th vector of the canonical basis of $\mathbb{R}^{m}$.

The control $\boldsymbol{u}^{*}(t)=\mathbf{0}$ is the optimal solution to problem (11) at time $t$ if and only if $\boldsymbol{p}(t) \boldsymbol{\Gamma}-\boldsymbol{k} \leq \mathbf{0}$. Otherwise, the value of the objective function of the problem (11) at the vertex $\boldsymbol{u}^{(j)}$ is

$$
\boldsymbol{p}(t) \gamma_{\cdot j}-b
$$

and then the control $\boldsymbol{u}^{\left(j^{*}\right)}$ is an optimal solution to problem (11) at time $t$ if and only if

$$
j^{*} \in \arg \max _{j} \boldsymbol{p}(t) \boldsymbol{\gamma}_{\cdot j} \cdot
$$

We have obtained that an optimal solution requires that at any time $t$ either the event organizer does not advertise, or he advertises using only media which solve the choice condition (22); if only one medium is used, say the $j$ th, then this is done at level $b / k_{j}$ (i.e. $\left.u_{j}=b / k_{j}\right)$.

Remark: The equation (22) provides the formulation of a medium choice prob$l e m$, but it is not directly useful, because in order to make it explicit we need to know the optimal state function $\left(\boldsymbol{G}^{*}(t), x^{*}(t)\right)$, as equations (15) and (14) witness. 
4.2 Constraints on medium activation levels only

Let $b>\boldsymbol{k} \overline{\boldsymbol{u}}$, so that we can ignore the global advertising cost rate constraint which is redundant.

The feasible region is the $m$-dimensional interval $\prod_{j=1}^{m}\left[0, \bar{u}_{j}\right]$ with the $2^{m}$ vertices

$$
\sum_{j \in \Sigma} \bar{u}_{j} \boldsymbol{e}_{j}, \quad \Sigma \subseteq\{1,2, \ldots m\}
$$

At an optimal solution $\boldsymbol{u}^{*}(t)$ to problem (11), and at any time $t \in[0, T]$, the constraint $u_{j} \leq \bar{u}_{j}$ must be active if $\boldsymbol{p}(t) \boldsymbol{\gamma}_{\cdot j}-k_{j}>0$, and inactive if $\boldsymbol{p}(t) \boldsymbol{\gamma}_{\cdot j}-k_{j}<0$; in the first case $u_{j}^{*}(t)=\bar{u}_{j}$, whereas in the latter $u_{j}^{*}(t)=0$. In other terms, an optimal solution to problem (11), at any time $t \in[0, T]$, is

$$
\boldsymbol{u}^{*}(t)=\sum_{j \in \Sigma(t)} \bar{u}_{j} \boldsymbol{e}_{j}, \quad \Sigma(t)=\left\{j \mid \boldsymbol{p}(t) \boldsymbol{\gamma}_{\cdot j}-k_{j}>0\right\}
$$

Remark: The equation (24) provides the formulation of a medium subset choice problem, and a similar observation as in the case of equation (22) applies here.

\section{Linear demand}

Let us consider the special choice of linear demand functions,

$$
f_{i}\left(G_{i}\right)=\beta_{i} G_{i}, \quad i=1,2, \ldots n,
$$

where the parameter $\beta_{i}>0$ is the marginal demand of goodwill in segment $i$ and we define the row vector $\boldsymbol{\beta}=\left(\beta_{1}, \beta_{2}, \ldots \beta_{n}\right)$. Then, the cumulative demand at time $t, x(t)$, is the solution to the Cauchy problem

$$
\dot{x}(t)=\boldsymbol{\beta} \boldsymbol{G}(t), \quad x(0)=0 .
$$


Under this assumption, the last term in the r.h.s. of the Hamiltonian definition (10) becomes

$$
q \sum_{i=1}^{n} f_{i}\left(G_{i}\right)=\boldsymbol{\beta} G
$$

The PMP conditions presented in Section 4 hold and, in particular, the $n$ dimensional adjoint function $\boldsymbol{p}(t)$ has the $i$ th component satisfying the equation (12), and hence $\boldsymbol{p}(t)$ must satisfy now the following linear differential equation with constant coefficients

$$
\dot{\boldsymbol{p}}(t)=\boldsymbol{p}(t) \operatorname{diag}(\boldsymbol{\delta})-q \boldsymbol{\beta} .
$$

We observe from the equations $(27),(13),(14)$ that the behaviour of the adjoint funcions $p_{i}(t)$ and the distinction among them depend only on segment specific features, and are independent of medium specific features. The Cauchy problem (27) and (13) has the explicit solution $\boldsymbol{p}(t)$ with positive and monotonically decreasing components

$$
p_{i}(t)=\frac{q \beta_{i}}{\delta_{i}}\left(1-e^{\delta_{i}(t-T)}\right), \quad i=1,2, \ldots n .
$$

Let us define the functions

$$
\varphi_{j}(t)=\frac{1}{q} \boldsymbol{p}(t) \boldsymbol{\gamma}_{\cdot j}=\sum_{i=1}^{n} \gamma_{i j} \frac{\beta_{i}}{\delta_{i}}\left(1-e^{\delta_{i}(t-T)}\right), \quad j=1,2, \ldots m,
$$

which are positive, continuous and monotonically decreasing, with $\varphi_{j}(T)=0$. The coefficients of the objective function of the problem (11), i.e.

$$
(\boldsymbol{p}(t) \boldsymbol{\Gamma}-\boldsymbol{k})_{j}=q \varphi_{j}(t)-k_{j}, \quad j=1,2, \ldots m
$$

are decreasing and continuous functions of $t$, moreover they are negative in a neighborhood of the final time $T$, as already known in the general case, because $q \varphi_{j}(T)-k_{j}=-k_{j}<0$. 
5.1 Constraint on global advertising cost rate only - linear demand

Let $b<\min _{j} k_{j} \bar{u}_{j}$, so that the medium activation level constraints are redundant. As $\boldsymbol{p}(t) \boldsymbol{\gamma}_{\cdot j}=q \varphi_{j}(t)$, the medium choice problem (22) can now be stated as

$$
j^{*} \in \arg \max _{j} \varphi_{j}(t)
$$

This is now an explicit formulation, as its solution depends exclusively on the control problem parameters, in contrast with what occurs in the nonlinear demand context, where the knowledge of the optimal state function $\left(\boldsymbol{G}^{*}(t), x^{*}(t)\right)$ is required.

In the special case that $\delta_{i}=\delta$ for all $i$, if there exists an optimal control which is not always equal to zero, then some optimal control is a vertex $(\neq \mathbf{0})$ of the feasible region of problem (11) and hence it is characterised by

$$
\boldsymbol{u}^{*}(t ; q)= \begin{cases}\left(b / k_{j^{*}}\right) \boldsymbol{e}_{\boldsymbol{j}^{*}}, & t \in\left[0, \tau_{j^{*}}(q)\right], \\ 0, & t \in\left(\tau_{j^{*}}(q), T\right]\end{cases}
$$

where

$$
\tau_{j^{*}}(q)=\varphi_{j^{*}}^{-1}\left(k_{j^{*}} / q\right)
$$

and

$$
j^{*}=\arg \max _{j} \boldsymbol{\beta} \gamma_{\cdot j}
$$

We can obtain an optimal solution to the advertising (control) problem by finding $q$ such that the condition (14), $q=R^{\prime}\left(x^{*}(T)\right)$, holds true with the state trajectories $\left(\boldsymbol{G}^{*}(t), x^{*}(t)\right)$ determined by the control $\boldsymbol{u}^{*}(t ; q)$. 
It is remarkable the fact that in this case the medium $j$ choice does not depend on time $t$.

5.2 Constraints on medium activation levels only - linear demand

Let $b>\boldsymbol{k} \overline{\boldsymbol{u}}$, so that the global advertising cost rate constraint is redundant. In view of the analysis of Section 4.2 , an optimal solution $\boldsymbol{u}^{*}(t ; q)$ to problem (11), at all times $t \in[0, T]$, is characterized by

$$
\boldsymbol{u}^{*}(t ; q)= \begin{cases}\bar{u}_{j}, & t \in\left[0, \tau_{j}(q)\right] \\ 0, & t \in\left(\tau_{j}(q), T\right]\end{cases}
$$

where

$$
\tau_{j}(q)= \begin{cases}\varphi_{j}^{-1}\left(k_{j} / q\right), & \text { if } \varphi_{j}(0)>k_{j} / q \\ 0, & \text { if } \varphi_{j}(0) \leq k_{j} / q .\end{cases}
$$

Finally, we can obtain an optimal solution to the advertising (control) problem by finding $q$ such that the condition $(14), q=R^{\prime}\left(x^{*}(T)\right)$, holds true with $\left(\boldsymbol{G}^{*}(t), x^{*}(t)\right)$ determined by the control $\boldsymbol{u}^{*}(t ; q)$.

The total effective advertising intensity directed to any market segment is a monotonically decreasing, stepwise function, with the value 0 in a neighborhood of the final time $T$. This qualitative result is in agreement with those obtained in [5], for seasonal products. The feature that the advertising efforts are decreasing is shared also by the results obtained in [8], although there the main motivation is the word-of-mouth communication process coupling the advertising one. 
The optimal control has a special bang-bang structure, characterized by the $m$ switch times $\tau_{j}, j=1, \ldots m$; these are advertising stopping times which we call advertising exposures. Let us denote by $x^{*}$ the number of sold tickets $x(T)$ associated with the optimal solution. Then, in view of the equations (14) and (28), the characterization of the optimal advertising exposures is, for all $j=1, \ldots m$ :

$$
\tau_{j}^{*}= \begin{cases}\varphi_{j}^{-1}\left(k_{j} / R^{\prime}\left(x^{*}\right)\right), & \text { if } \varphi_{j}(0)>k_{j} / R^{\prime}\left(x^{*}\right) \\ 0, & \text { if } \varphi_{j}(0) \leq k_{j} / R^{\prime}\left(x^{*}\right)\end{cases}
$$

We can make the information on the optimal solution more explicit in terms of exposures by integrating the motion equations. Let $\boldsymbol{G}(t ; \boldsymbol{\tau})=\boldsymbol{G}\left(t ; \tau_{1}, \ldots, \tau_{m}\right)$ be the unique solution to the motion equation (2) with

$$
\boldsymbol{w}(t)=\boldsymbol{\Gamma} \boldsymbol{u}^{*}(t ; q)=\sum_{j=1}^{m} \gamma_{\cdot j} u_{j}^{*}(t ; q)
$$

with switch times $\tau_{j}(q)$ as defined by equation (36); we have that

$$
\boldsymbol{G}(t ; \boldsymbol{\tau})=e^{-\operatorname{diag}(\boldsymbol{\delta}) t} \boldsymbol{G}^{\mathbf{0}}+\sum_{j=1}^{m} \bar{u}_{j} e^{-\operatorname{diag}(\boldsymbol{\delta}) t} \int_{0}^{\min \left\{\tau_{j}, t\right\}} e^{\operatorname{diag}(\boldsymbol{\delta})(s)} \boldsymbol{\gamma}_{\cdot j} d s
$$

After integrating the motion equation (6), we obtain that the number of tickets sold in the interval $[0, T]$ is

$$
x(T ; \boldsymbol{\tau})=x(T ; \mathbf{0})+\sum_{i=1}^{n} \frac{\beta_{i}}{\delta_{i}^{2}} \sum_{j=1}^{m} \bar{u}_{j} \gamma_{i j}\left(\delta_{i} \tau_{j}+e^{-\delta_{i} T}-e^{\delta_{i}\left(\tau_{j}-T\right)}\right),
$$

where

$$
x(T ; \mathbf{0})=\sum_{i=1}^{n} \frac{\beta_{i} G_{i}^{0}}{\delta_{i}}\left(1-e^{-\delta_{i} T}\right)
$$

is the number of tickets the organizer can sell without any advertising effort. 


\section{Conclusion}

In this paper we have studied the problem of advertising a social event in a segmented market using different media. Assuming additive advertising effects on goodwill evolution, and goodwill concave demand rate, we have formulated the optimal control problem of maximizing the profit from the sale of tickets for the entertainment event. We have observed that the Pontryagin Maximum Principle conditions involve the discussion of a family of linear programming problems, with time-varying objective functions. This is the framework for the discussion of existence of an optimal solution and for its characterization.

\section{Acknowledgements}

The research was supported by the Italian Ministry of University and Research, the University of Padova and Ca' Foscari University of Venice.

\section{References}

1. A. Buratto And L. Grosset, A communication mix for an event planning: a linear quadratic approach, Central European Journal of Operations Research, 14 (2006), pp. $247-259$

2. A. Buratto, L. Grosset, And B. Viscolani, Advertising a new product in a segmented market, European Journal of Operational Research, 175 (2006), pp. 1262-1267.

3. — Advertising channel selection in a segmented market, Automatica, 42 (2006), pp. $1343-1347$.

4. M. EASEY, Fashion marketing, Wiley, Oxford, 2007. 
5. D. Favaretto And B. Viscolani, Advertising and production of a seasonal good for a heterogeneous market, 4OR - A Quarterly Journal of Operations Research, 8 (2010), pp. $141-153$

6. J. Huang, M. Leng, And L. LiAng, Recent developments in dynamic advertising research, European Journal of Operational Research, 220 (2012), pp. 591-609.

7. G. Iyer, D. Soberman, And J. M. Villas-Boas, The targeting of advertising, Marketing Science, 24 (2005), pp. 461-476.

8. S. Jørgensen, P. M. Kort, And G. Zaccour, Advertising an event, Automatica, 42 (2006), pp. 1349-1355.

9. —_ Optimal pricing and advertising policiesfor an entertainment event, Journal of Economic Dynamics and Control, 33 (2009), pp. 583-596.

10. S. Jørgensen And G. Zaccour, Differential Games in Marketing, Kluwer Academic Publishers, Boston, 2004.

11. - A survey of game-theoretic models of cooperative advertising, European Journal of Operational Research, 237 (2014), pp. 1-14.

12. P. Kotler, Marketing Management, 11th Edition, Prentice-Hall, Upper Saddle River, 2003.

13. M. Nerlove And K. J. Arrow, Optimal advertising policy under dynamic conditions, Economica, 29 (1962), pp. 129-142.

14. A. Seierstad And K. Sydsaeter, Optimal Control Theory with Economic Applications, North-Holland, Amsterdam, 1987.

15. B. Viscolani, Advertising decisions for a segmented market, Optimization, 58 (2009), pp. $469-477$.

16. M. Winchester And G. LeEs, Do radio stations in New Zealand target successfully?, Australasian Marketing Journal, 21 (2013), pp. 52-58. 